\title{
A Model for Electron/Ion \\ Recombination in Ionization Chambers
}

\author{
William C. Sailor
}

\section{DISCLAIMER}

This report was prepared as an account of work sponsored by an agency of the United States Government. Neither the United States Government nor any agency thereof, nor any of their employecs, makes any warranty, express or implied, or assumes any legal liability or responsibility for the accuracy, completeness, or usefulness of any information, apparatus, product, or process disclosed, or represents that its use would not infringe privately owned rights. Reference herein to any specific commercial product, process, or service by trade name, trademark, manufacturer, or otherwise does not necessarily constitute or imply its endorsement, recommendation, or favoring by the United States Government or any agency therenf. The views and opinions of authors expressed herein do not necessarily state or reflect those of the United States Government or any agency thereof.

\section{MASTER}




\title{
A MODEL FOR ELECTRON/ION RECOMBINATION IN IONIZATION CHAMBERS
}

\author{
by
}

William C. Sailor

\begin{abstract}
The recombination of free electrons and positive ions along charged particle tracks in gases has been modeled using electron transport equations, which assume homogeneous distributions in the vicinity of the tracks. The equations include space charge terms, which have been neglected in previous models. A formula for the electron yield as a function of detector applied potential is obtained from a perturbation solution valid when the ratio of the Debye length to the charge column radius is larger than unity. When this ratio is very large, the formula reduces to that of previous models. Fulse height measurements in a ${ }^{3} \mathrm{He}$ ionization chamber indicate $2 \%$ to $30 \%$ losses to recombination which vary with applied field, particle type, and energy. Using reasonable values for the electron transport coefficients, the calculated loss of signal to recombination is generally in agreement with experiment, but the variation with applied bias is stronger in the experiment.
\end{abstract}

\section{Introduction}

Recombination in gas chambers where free electrons are the charge carriers has rarely been discussed in the literature, and there is no model for an increased recombination rate with increasing gas density due to space charge effects. Unexpectedly large signal losses to recombination were discovered ${ }^{1,2}$ during the energy calibration of a commercial ${ }^{3} \mathrm{He}$ neutron ionization chamber, the seforad FNS-13. The $5 \mathrm{~cm}$ diameter gridded 
cylindrical chamber uses 6 atm ${ }^{3} \mathrm{He}, 3$ atm argon, and 1 atm $\mathrm{CH}_{4}$ for its fill gas, and electric fields typically of $50 \mathrm{~V} / \mathrm{cm}$-atm. In the pulse height distributions resulting from 0.5 to $3 \mathrm{MeV}$ monoenergetic neutrons, it was found that the pulses from elastic scattering on ${ }^{3} \mathrm{He}$ were smaller by 12 $25 \%$ than the protons and tritons from ${ }^{3} \mathrm{He}(n, p)$ events per unit ionization energy. Deficits in pulse height of 5 to $10 \%$ for ${ }^{3} \mathrm{He}$ or $\propto$ particles versus protons have been reported elsewhere 4,5 and have usually been explained in terms of differing $W$-values (mean energy loss per ion pair formed). However, there are many experiments ${ }^{6}$ which show that $w$ does not vary with velocity or particle type by more than a few percent for particle energies over $100 \mathrm{keV}$. Many gas chambers are operated at about atmospheric pressure; however, neutron detectors of ten use elevated pressures to increase detection efficiency. The sizable loss of free electrons to recombination in the FNS-1 chamber is presumably due to the elevated pressure and higher charge density along the ${ }^{3} \mathrm{He}$ tracks.

A theory of recombination of positive and negative ions along particle tracks, developed by Jaffe 7,8 , incorporated diffusion away from the track, as $w \in l l$ as drift according to the applied field in the detector. Recombination occurred in the region of overlap of the two clouds, which moved in opposite directions from one another. The model has been applied to gases, liquified rare gases ${ }^{9-13}$, organic solids ${ }^{14}$, and semiconductors ${ }^{15-17}$. It has been used for all types of ionizing radiation. Lea ${ }^{18}$ claimed that for beta rays, recombination occurs mostly in localized clusters, which he modeled in a manner similar to Jaffe's, except with spherical geometry. Kramersis modified Jaffe's model for liquified gases such as helium, where the assumed temperature of the charge carriers is very low, to exclude difiusion. Messenger ${ }^{20}$ modified Jaffe's theory for very high ionization densities along ion tracks in irradiated semiconductors by using the ambipolar diffusion constant for the excess charge carriers, which thereby considers the electrostatic forces between the electron and positive ion clouds.

In this paper a model of electron/ion recombination along particle tracks applicable to gas chambers is developed, which gives the fraction of electrons escaping recombination as a function of applied field. Space charge effects, for moderately high charge densities, are included. 


\section{Modeling Considerations}

No one recombination theory can cover the entire range of particle types and energies interacting with all types of materials. The distribution of primary electron energies is a function of the particle charge and velocity and of the cross sections in the gas, and so the relative importance of diffusion, drift, and spaca charge effects may vary greatly. Even the geometry of the charge distributions will depend on the particle energy. For low dE/dx tracks, much of the ionization may form into "spurs" and "blobs" of electrons and ions. High energy particles produce $\delta$-rays, which in turn are low dE/dx tracks.

Slow, heavy charged particles slowing down in a dense rare gas produce a spectrum of primary electrons which are distributed somewhat uniformly around the track. Some of the hot electrons produce further ionization, while cooling through inelastic processes. During this period, the electrons are confined to a characteristic radius around the track, $b$, and have a time-dependent temperature kT. If the charge density is high enough, a weak plasma of electrons and ions forms. On a longer time scale, a fraction of the electrons recombine with positive ions. The remainder diffuse away from the track, and drift in the applied field of the detector. When the electrons have moved a sufficient distance from the ions, the recombination process will terminate provided there is a sufficiently strong applied field. In an ionization chamber the signal is proportional to the number of electrons which have escaped recombination.

Assuming a recombination process of the form $d n / d t=-\alpha n n^{+}$, where $n$ and $n^{+}$ are the electron and positive ion concentrations, respectively, and $\alpha$ is the recombination coefficient, it is implied that for a $10 \%$ recombination loss, $\propto n \Delta t \approx 10^{-1}$. The amount of time, $\Delta t$, required for the electrons to escape the positive ions will be determined by the column radius $b$, the electron mobility $\mu$, and diffusivity $D$. If a weak plasma forms, $\Delta t$ will be increased because of the tendency of plasmas to shield themselves from applied fields. To form a plasma the Debye length $\left(\lambda_{D}=\sqrt{\mathrm{kT/4 \pi nq_{e }}}\right)$ must be smaller than b. In addition, the "Plasma Parameter" $N_{D}=n \times 4 \pi \lambda_{D} 3 / 3$ (a measure of the homogeneity of the charge distribution) must be much greater than unity.

The recombination process which determines $\alpha$ will depend on the composition, pressure, and temperature of the gas ${ }^{21-23}$. Measurements of the 
pressure dependence of electron-ion recombination showed ${ }^{24}$, for $\mathrm{NH}_{3}, \alpha$ increases to a maximum value at 700 torr, and then decreases as the pressure was raised further. The increases are attributed $25-27$ to the enhancement of dissociative recombination by collisions with neutral molecules (essentially a three body process), which is ultimately limited by the electron mobility at the highest pressures, where the Langevin recombination coefficient formula holds. This is taken as the explanation for the ultimate decrease in the $\mathrm{NH}_{3}$ coefficient after reaching its peak value.

A $1.9 \mathrm{MeV}{ }^{3} \mathrm{He}$ nucleus (characteristic from elastic scattering from a $2.6 \mathrm{MeV}$ neutron) slowing in the FNS-1 fill gas will produce primary electrons with an average energy (assuming Rutherfors scattering) of about $700 \mathrm{eV}$. Over the next $10^{-12} \mathrm{sec}$ the electrons produce between 0 and 10 further free electrons each. At this point the electrons should be characteristically confined to a radius $b=10^{-4} \mathrm{~cm}$, based on an electron scattering ionization mean free path of about $10^{-5} \mathrm{~cm}^{28}$. After falling below the excitation threshold, the electrons have a spread in energies from 0 to $10 \mathrm{eV}$. The electron temperature when in equilibrium with a $100 \mathrm{~V} / \mathrm{cm}$-atm applied field is about $0.34 \mathrm{eV}^{29}$, and so the electrons will continue to cool until reaching this temperature. The time required for this is determined by the $\mathrm{CH}_{4}$ vibrational excitation cross sections ${ }^{30}$ (about $10^{-16} \mathrm{~cm}^{2}$ ) giving $\Delta \mathrm{t}_{\mathrm{cool}} \approx 10^{-11} \mathrm{sec}$. The random walk distance for the electrons during this time is about $10^{-4} \mathrm{~cm}$, essentially equal to the column radius; $i$. e. the time scale for diffusion is the same as that for cooling. The time scale for the electrons to drift across a column diameter will depend on the temperature, but if $k T=1 \mathrm{eV}$, the drift time is an order of magnitude larger than $\Delta t_{\text {cool. Also under the }}$ assumption that $k T=1 \mathrm{eV}$, the Debye length $\lambda_{D} \approx 2 \mathrm{~b}$ and $N_{D}=30$.

The modeling of the system is complicated because several time scales and distance scales are of the same order of magnitude. A rigorous calculation (e.g. Monte Carlo) would be difficult because of the lack of cross section and recombination coefficient data. In order to simplify these calculations, no time dependence for the temperature is assumed. Electron and positive ion distributions are modeled as homogeneous charge distributions which follow transport laws. The time dependences of the diffusion constant, mobility, and Debye length are neglected. 


\section{Electron Transport Equations}

The positive ions are assumed immobile, and to exist in a spacial distribution

$$
n^{+}(x, y)=n^{0} e^{-\left[x^{2}+y^{2}\right] / b^{2}}
$$

where $D$ is an effective column radius, and $n^{0}$ is a normalizing concentration

$$
n^{0}=(d E / d x) /\left(\pi b^{2} W\right)
$$

The electrons are mobile, have a time dependent distribution $n(x, y, t), a$ diffusion constant, $D$ and a mobility $u$, and an initial distribution

$$
n(x, y, t=0)=n^{0} e^{-\left[x^{2}+y^{2}\right] / b^{2}} \text {. }
$$

The diffusion constant and mobility are taken as time independent. The continuity equation, including recombination, and a Fick's Law assumption for the current is stated as:

$$
\partial n / \partial t-D \nabla^{2} n+\mu \nabla \phi \cdot \nabla n+\mu n \nabla^{2} \phi=-\alpha n n^{+}
$$

where $\mu$ is the mobility (taken as positive) and $\phi$ is the potential. For the potential there is Poisson's equation

$$
\nabla^{2} \phi=-4 \pi q_{e}{ }^{\left(n^{+}-n\right)}
$$

where $q_{e}$ is the fundamental charge. The boundary conditions on the potential are that it becomes the specified potential at the walls of the chamber. Because the dimensions of the chamber are several orders of magnitude larger than the characteristic dimension of the charge distribution, the potential is considered as the sum of two potentials, $\phi=\phi_{c}+\phi_{s}$, where $\phi_{C}$ meets the boundary conditions at the walls of the chamber (i.e. the applied potential), and $\phi_{S}$ (due to space charge) vanishes at infinity.

The assumed geometry between the track and the walls of the chamber is shown in Fig. la and between the track and the normal component of the 


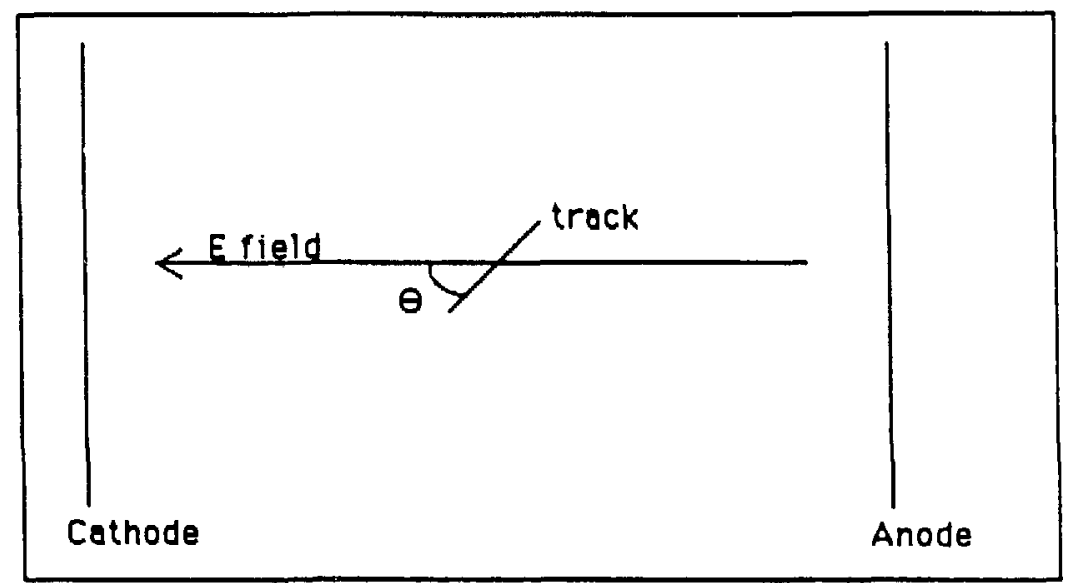

Fig. Ia. Orientation of track and detector field lines.

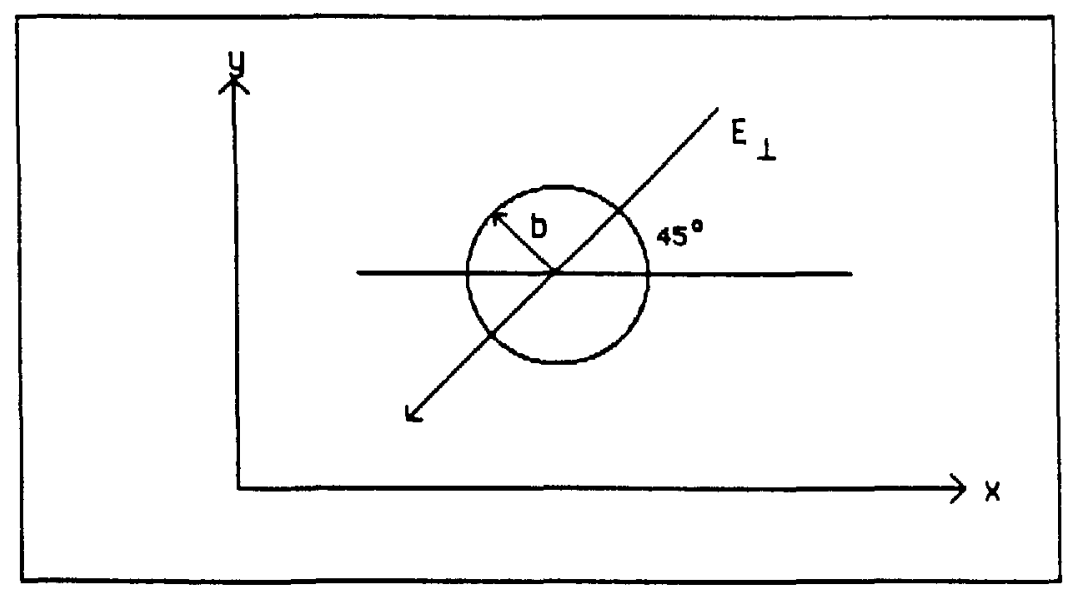

Fig. 10. Orientation of track with respect to $E_{\perp}$.

The track comes out of the page. The column radius is $b$. 
field, $E_{\perp}$, in Fig. 1b. The component of the field normal to the track has magnitude $E_{\perp}$, and points in the negative $x$ and negative $y$ direction, so that the electrons drift in the positive direction. The $x$ and $y$ components (in the track coordinate system) of the imposed field are

$$
E_{c, x}=E_{c, y}=-E_{\perp} / \sqrt{2}
$$

A change to dimensionless variables is made:

$$
\begin{array}{ll}
c=n / n^{0}, & \Phi=\phi /\left(4 \pi b^{2} q_{\left.e^{n^{0}}\right),}\right. \\
\eta=y / b, & \xi=x / b, \quad \tau=D t / b^{2} .
\end{array}
$$

The rearranged Equations 4 and 5 are then

$$
\begin{aligned}
& \partial c / \partial t-\nabla^{2} c+v \nabla c+g\left\{\nabla \Phi \cdot \nabla c-c\left(c^{+}-c\right)\right\}=-P c c^{+} \\
& \nabla^{2} \Phi=-\left(c^{+}-c\right)
\end{aligned}
$$

the initial condition and positive ion concentration are:

$$
c(\xi, \eta, 0)=c^{+}(\xi, \eta)=e^{-\left(\xi^{2}+\eta^{2}\right)}
$$

and the boundary condition on $\Phi$ is that it vanishes at infinity. The dimensionless groups $v, g$, and $P$ are defined as:

$$
\begin{aligned}
& v=\frac{b \mu E_{\perp} / \sqrt{2}}{D}>0 \\
& P=\frac{\alpha(d E / d x)}{\pi D W}>0 \\
& g=\frac{4 \pi \mu q_{e} n_{0} b^{2}}{D}=\frac{b^{2}}{\lambda_{D}^{2}}>0
\end{aligned}
$$


where the second equality in 13 was obtained using the Einstein relation:

$$
\mu / D=q_{e} / k T
$$

The electron transport equation and also the fraction of electrons recombining will be a function only of these three variables $V, P$, and $g$. When the recombination coefficient is given by the Langevin formula, $\alpha_{L}=$ $4 \pi \mu q_{e}$, then $P=g$. Both groups are independent of the radius $b$, because the Debye length is proportional to $b$. All three groups increase with decreasing temperature; as the electrons cool, the electrons have a greater mobility and so the Langevin recombination coefficient increases as well as the dimensionless drift velocity.

\section{Perturbation Solution}

The recombination coefficient $P$ is set temporarily to zero and a perturbation solution to the system of Equations 8 and 9 is sought for small values of $\mathrm{g}$. After the solution is found, it is adjusted for larger values of P. Therefore, Equation 8 is restated as:

$$
L c=g\left(-\nabla \Phi \cdot \nabla c+c\left(c^{+}-c\right)\right\}
$$

where the linear operator $L$ is given by:

$$
L=\frac{\partial}{\partial \tau}-\left(\frac{\partial^{2}}{\partial \xi^{2}}+\frac{\partial^{2}}{\partial \eta^{2}}+v\left(\frac{\partial}{\partial \xi}+\frac{\partial}{\partial \eta}\right)\right.
$$

The solution to this equation is assumed to be

$$
c=c_{0}+g c_{1}+g^{2} c_{2}+\ldots g<1
$$

where the $c_{0}$ solution meets the initial condition and the $c_{1}$ and higher solutions meet homogeneous initial conditions. The system is formed: 


$$
\begin{array}{ll}
L c_{0}=0 & c_{0}(\xi, \eta, 0)=c(\xi, \eta, 0) \\
L c_{1}=g \Psi_{1}(\xi, \eta, \tau) & c_{1}(\xi, \eta, 0)=0 \\
\dot{v} & \dot{\cdot} \\
\dot{L} c_{k}=g \Psi_{k}(\xi, \eta, \tau) & c_{k}(\xi, \eta, 0)=0
\end{array}
$$

where the functions

$$
\Psi_{k+1}(\xi, \eta, \tau)=-\left(\partial c_{k} / \partial \xi\right)\left(\partial \Phi_{k} / \partial \xi\right)-\left(\partial c_{k} / \partial \eta\right)\left(\partial \Phi_{k} / \partial \eta\right)+c_{k} c^{+}-c_{k}{ }^{2}
$$

and the $\Phi_{k}$ are obtained from the solution of Equation 8 while $c_{k}$ is substituted into the right-hand side. For the $k=0$ solution, i.e. $g=0$, the electron transport problem is a standard convection/diffusion problem, which is solved using the Green's function:

$$
G\left(\xi, \xi^{\prime}, \eta, \eta^{\prime}, \tau\right)=\frac{e^{-\left[\left(\xi-\xi^{\prime}-v \tau\right)^{2}-\left(\eta-\eta^{\prime}-v \tau\right)^{2}\right] / 4 \tau}}{4 \pi \tau}
$$

The solution is:

$$
c_{0}(\xi, \eta, \tau)=\mathrm{e}^{-\left[(\xi-v \tau)^{2}+(\eta-v \tau)^{2}\right] /(1+4 \tau) /(1+4 \tau)}
$$

The fraction of free electrons escaping recombination could be calculated from this solution and a Jaffe-like formula would result. Instead, the $c_{1}$ term is found at this point.

In order to find this solution, the right-hand side of Equation 19 is first found for $k=0$. The cylindrical symmetry for the electron charge distribution about the line $\xi=\eta=v \tau$, under the assumption that the particle track is many order of magnitudes longer than it is wide, and that the space charge fields have not perturbed the charge distributions significantly, is exploited. About the line $\xi=\eta=0$ the positive ion cloud is also cylindrically symmetric. The integral form of Gauss's law is then used 
separately on the electrons and positive ions, in order to obtain the gradients of The resulting expression for $\Psi_{1}$ is too lengthy to be given here, but it does have an analytical form. The solution to the equation $L c_{1}=\Psi_{i}$ with a homogeneous initial condition is given by:

$$
c_{1}(\xi, \eta, \tau)=\int_{0}^{\tau} \int_{-\infty}^{\infty} \int_{-\infty}^{\infty} \Psi_{1}\left(\xi^{\prime}, \eta^{\prime}, \tau^{\prime}\right) G\left(\xi, \xi^{\prime}, \eta, \eta^{\prime}, \tau-\tau^{\prime}\right) d \xi^{\prime} d \eta^{\prime} d \tau^{\prime}
$$

\section{Fraction of Electrons Recombining}

It can be shown that the fraction of electrons recombining is given in a general case as

$$
t_{r}=\int_{0-\infty}^{\infty} \int_{-\infty}^{\infty} \int P c(\xi, \eta, \tau) c^{+}(\xi, \eta) d \xi d \eta d \tau
$$

which here is (assuming $\alpha=\alpha_{L}$, or $P=g$ ):

$$
f_{r}=g F_{0}(v)+g^{2} F_{1}(v)+\ldots,
$$

where,

$$
F_{k}(v)=\int_{0}^{\infty} \int_{-\infty}^{\infty} \int_{-\infty}^{\infty} c_{k} c^{+} d \xi d \eta d \tau
$$

The $c_{0}$ and $c_{1}$ solutions and $c^{+}$concentration are substituted into Equation 25 and the results integrated over their spacial coordinates analytically. The result is:

$$
\begin{aligned}
& F_{0}(v)=\int_{0}^{\infty} e^{-[2 v \tau /(1+4 \tau)][(2+4 \tau) v \tau-1] /[2+4 \tau]} d \tau /(2+4 \tau), \\
& F_{1}(v)=\int_{0}^{\infty} \int_{0}^{\tau} G_{3}\left(\tau^{\prime}, \tau-\tau^{\prime}\right) d \tau^{\prime} d \tau
\end{aligned}
$$


where,

$$
G_{3}\left(\tau^{\prime}, \tau-\tau\right)=\frac{v \tau^{\prime}\left[f_{1}\left(A_{1}, B_{1}, C_{1}\right)-r_{1}\left(A_{2}, B_{2}, C_{2}\right)\right]+\left[2+4 \tau^{\prime}\right] f_{0}\left(A_{2}, B_{2}, C_{2}\right)-2 f_{0}\left(A_{3}, B_{3}, C_{3}\right)}{\left(1+4 \tau^{\prime}\right)^{2}\left[1+4\left(\tau-\tau^{\prime}\right)\right]}
$$

$$
f_{0}(A, B, C)=\frac{\pi e^{2\left[B^{2}-A C\right] / A}}{A}
$$

$f_{1}(A, B, C)=\sqrt{2 \pi / A} e^{2\left(B^{2}-A C\right) / A} f_{2}(B \sqrt{2 / A})$,

$f_{2}(\beta)=-2 e^{-\beta^{2}} \int_{0}^{1} e^{\beta^{2} v^{2}} \operatorname{erf}[\beta v] \frac{v d v}{\sqrt{1-v^{2}}}$

$$
\begin{aligned}
& A_{1}=1 /\left(1+4 \tau^{\prime}\right)+1 /\left[1+4\left(\tau-\tau^{\prime}\right)\right], \\
& A_{2}=1+1 /\left(1+4 \tau^{\prime}\right)+1 /\left[1+4\left(\tau-\tau^{\prime}\right)\right], \\
& A_{3}=2 /\left(1+4 \tau^{\prime}\right)+1 /\left[1+4\left(\tau-\tau^{\prime}\right)\right], \\
& B_{1}=-v \tau /\left(1+4 \tau^{\prime}\right)+v\left(\tau-\tau^{\prime}\right) /\left[1+4\left(\tau-\tau^{\prime}\right)\right], \\
& B_{2}=-v \tau^{\prime} /\left(1+4 \tau^{\prime}\right)+v(\tau-\tau) /\left[1+4\left(\tau-\tau^{\prime}\right)\right], \\
& B_{3}=-2 v \tau^{\prime} /\left(1+4 \tau^{\prime}\right)+v\left(\tau-\tau^{\prime}\right) /\left[1+4\left(\tau-\tau^{\prime}\right)\right], \\
& C_{1}=v^{2} \tau^{\prime 2} /\left(1+4 \tau^{\prime}\right)+v^{2}\left(\tau-\tau^{\prime}\right)^{2} /\left[1+4\left(\tau-\tau^{\prime}\right)\right], \\
& C_{2}=v^{2} \tau^{\prime 2} /\left(1+4 \tau^{\prime}\right)+v^{2}\left(\tau-\tau^{\prime}\right)^{2} /\left[1+4\left(\tau-\tau^{\prime}\right)\right],
\end{aligned}
$$

and,

$$
C_{3}=2 v^{2} \tau^{\prime 2} /\left(1+4 \tau^{\prime}\right)+v^{2}\left(\tau-\tau^{\prime}\right)^{2} /\left[1+4\left(\tau-\tau^{\prime}\right)\right]
$$


The integrals in Equations 26 and 27 were evaluated numerically, using 64 point Gauss-Legendre integration over the $\tau$ variables, with upper limits of $5 / v$ and $\max \left(10 / v, 10 / v^{2}\right)$, respectively. The results were checked against 96 points integration. For the $\tau$ ' veriable in Equation 27 an adaptive Gauss-Legendre method (DGAUSS from the Cern Mathematicai Software Library KERNLIB) was used. The results are shown in Fig. 2.

(The value of the $f_{2}$ function was tabulated at 200 values of $0<\beta<9$, by using adaptive Gauss-Legendre integration to a relative precision of better than $10^{-3}$. Newton's interpolation method with first and second differences was then used from the values in the table. Since the function is odd, $f_{2}(-\beta)$ $=-f_{2}(\beta)$ was used for negative $B$. For $\beta>9$ an asymptotic approximation was used.)

\section{Extension to Higher Fractions Recombined}

The analysis until now depends on the assumption that the fraction of electrons recombining is small, as well as the assumption that $\mathrm{g}$ is small. We desire a formula that extends to higher $f_{r}$, reduces to Equation 24 for small $f_{r}$, and also reduces to a Jaffe-like form for $g=0$. Such a formula is stated in terms of the fraction unrecombined $f_{u}$ as:

$$
f_{u}=1-f_{r}=\left(1+g\left[F_{0}(v)+g F_{1}(v)\right]\right)^{-1} \text {. }
$$

The formula gives the desired asymptotic behavior, i.e. $f_{u}(v) \rightarrow 0$ as $v \rightarrow 0$, and $f_{u}(v) \rightarrow 1$ as $v \rightarrow \infty$. For $f_{r}<0.1$ it is nearly equivalent to Eq. 24. Plots of the function are given in the next section with the experimental results.

\section{Experimental Pulse Height Variation With Applied Potential}

The applied biases to the grid and anode of the FNS-1 detector were varied while the detector was irradiated with neutrons from the $D(D, n)$ reaction and the movement in the location of the ${ }^{3} \mathrm{He}$ recoil edge in the pulse height spectrum was observed. During the experiment it is desired that the electric field lines in the detector maintain a constant profile, which implies for a cylindrical detector that the anode potential is varied in a proportion which kueps the ratio of the grid to anode potential a constant, i.e. $850 \mathrm{~V} / 3000 \mathrm{~V}$. 
Neutrons of $2.59 \pm 0.03 \mathrm{MeV}$ were obtained with a Karnan Sciences neutron generator, which was operated with an accelerating voltage of $150 \mathrm{kV}$ with a current of about $2 \mathrm{~mA}$. Targets were titanium deuteride on thick copper backings. The detector was located $33 \mathrm{~cm}$ from the target at $74 \pm 2^{\circ}$ away from a straight path. A Nuclear Data Corp. ND-65 served as a multichannel analyzer to collect the pulse height data.

The family of curves shown in Fig. 3 is the result of 1 plot for each applied bias, the highest bias for the top curve, and in descending order to the bottom. The channels 79,157 , and 309 are the location of the centroid of the thermal peak, of the edge of the ${ }^{3} \mathrm{He}$ elastic scattering continuum, and of the full-energy peak for the $\Phi_{g}=850 \mathrm{~V}$ run. The location of the ${ }^{3} \mathrm{He}$ edge shifts leftwards, and the edge becomes broader with decreasing voltage. The full-energy peak tends to become broader with decreasing voltage, especially the low-energy tail. The location of the centroid changed by only a few channels.

Table 1 gives the channel number of the centroid of the edge for each response (determined by hand drawn curves through the data). The uncertainty is considered to be \pm 1 channel. The prediction from the kinematics of the neutron elastic scattering reaction on ${ }^{3} \mathrm{He}$ is that the edge would lie in channel $188 \pm 1$, under the assumption that the $W$-value for the ${ }^{3} \mathrm{He}$ nucleus is exactly the same as that for the proton. In view of theoretical arguments and most experimental observations this is the correct assumption. The greatest difference found experimentally between $W_{\alpha}$ and $W_{p}$ was $8 \%$; under this assumption channel $175 \pm 1$ represents the maximum pulse height. The zero pulse height channel is $11 \pm 1$.

The data points in Fig. 4 show the variation in the fraction of full signal for the ${ }^{3} \mathrm{He}$ recoil edge with applied potential for the two assumptions regarding $w$. The family of curves shown as solid lines are from Equation 33 , with the assumptions used to determine the dimensionless group v: 
Fig. 2. The Functions $F_{0}(v)$ and $F_{1}(v)$

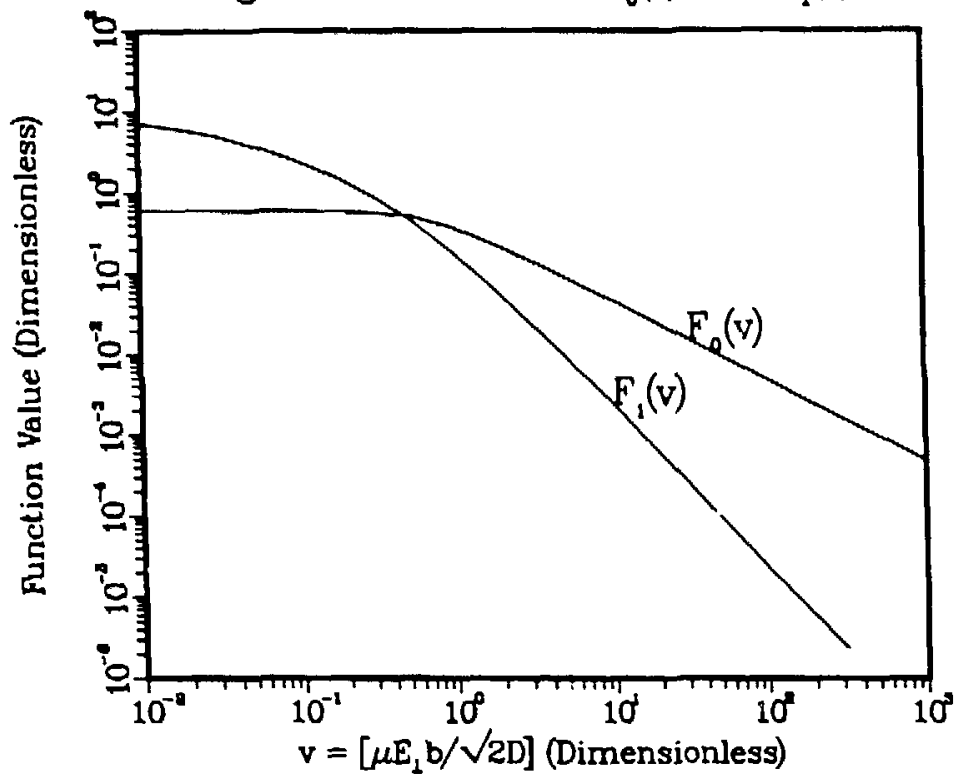

Fig. 3. FNS-1 Pulse Height Distribution From 2.58 MeV D(D,n) Neutron Source

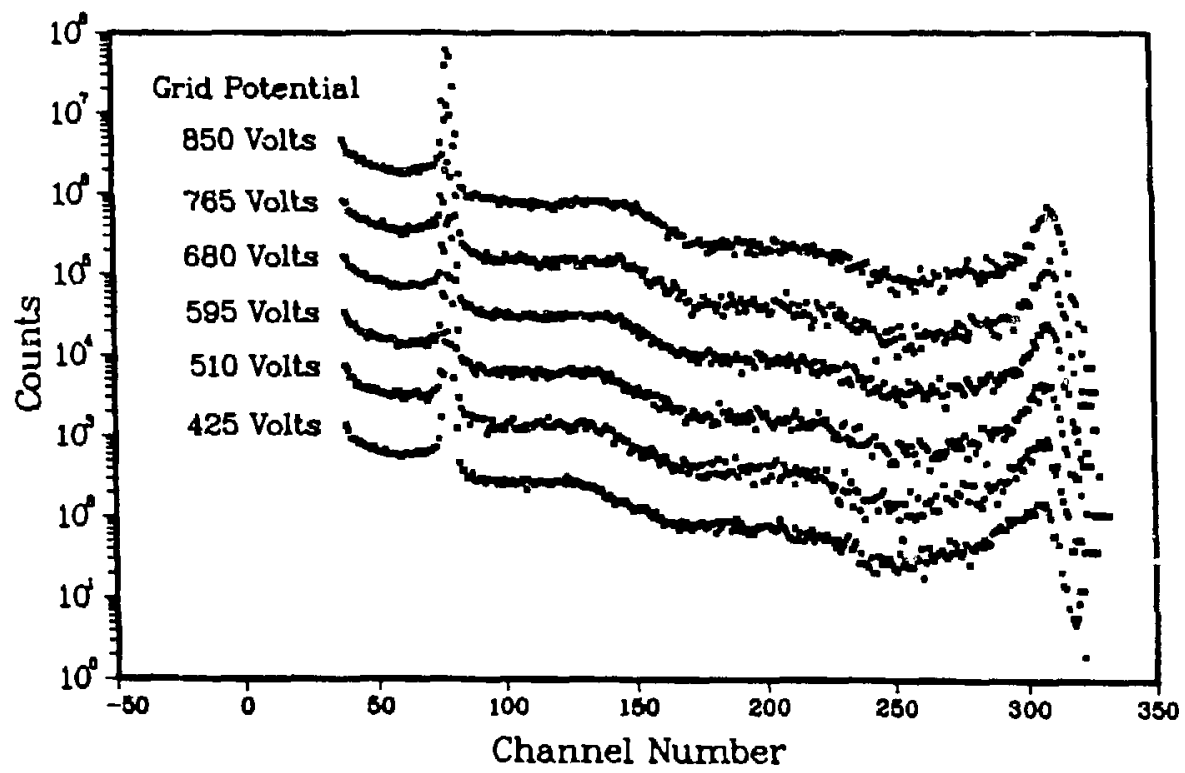


Table 1. Variation in Location of ${ }^{3} \mathrm{He}$ Recoil Edge With Bias in $D(D, n)$ Neutron Experiment.

\begin{tabular}{lll}
$\begin{array}{l}\text { Grid } \\
\text { Voltage, } \Phi_{g}\end{array}$ & $\begin{array}{l}\text { Anode } \\
\text { Voltage, } \Phi_{\mathrm{a}}\end{array}$ & $\begin{array}{l}\text { Centroid } \\
\text { Channel Number }\end{array}$ \\
\cline { 2 - 3 } & & \\
\hline 850 & 3000 & 157.1 \\
765 & 2700 & 155.1 \\
680 & 2400 & 150.7 \\
595 & 2100 & 149.1 \\
510 & 1800 & 143.5 \\
425 & 1500 & 141.9
\end{tabular}

$$
\begin{aligned}
& \mu=1.3 \times 10^{3}(\mathrm{~cm} / \mathrm{sec}) /(\text { Volt } / \mathrm{cm}) \\
& D=100 \mathrm{~cm}^{2} / \mathrm{sec} \\
& D=1 \times 10^{-4} \mathrm{~cm}
\end{aligned}
$$

The mobility and diffusion constant are calculated from a simplified method given in Ref. 29 , where it is assumed that the electrons are at an equilibrium temperature $(\approx 0.34 \mathrm{eV}$ ) in the electric field of the detector. The component of the electric field normal to the particle track varies with event to event. This variation gives the recoil edge its broad shape. An average value of the electric field between the grid and cathode (with radii $r_{g}$ and $r_{c}$, respectively) is taken to be:

$$
\left\langle E_{\perp}\right\rangle=\frac{4 \Phi_{g}}{\pi\left(r_{c}+r_{g}\right) \ln \left(r_{c} / r_{g}\right)}
$$


The value of $\mathrm{g}$ is 0.20 when using the following assumptions:

$$
\begin{aligned}
& D / \mu=k T / q_{e}=1 \text { Volt } \\
& d E / d x=8 \mathrm{MeV} / \mathrm{cm} \\
& W=25 \mathrm{eV} / \text { ion pair }
\end{aligned}
$$

The curves for $g=0.10$ and $g=0.30$ bracket the data. The model predicts the correct fraction recombined, for the $W_{\alpha}=1.08 W_{\text {proton }}$ case, to within about 30x. For the $w_{\alpha}=W_{\text {proton }}$ assumption, the model with $g=0.30$ is within $20 x$ in predicting $f_{r}$. The slope of the model prediction is not as steep as experiment, probably because of the neglect of higher order terms in Equation 29. The $g=0$ curve, on the other hand, shows almost no slope in this range of potentials, indicating that without the space charge effects, variation in pulse height with applied potential should not be expected, nor should the fraction recombined be so large. The critical field (where $v=1$ ) from this set of constants is

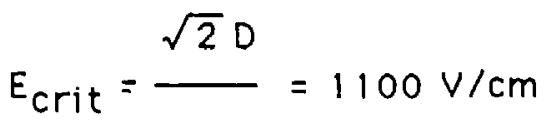

$\mu b$

The grid bias which gives $\left\langle E_{\perp}\right\rangle=E_{\text {crit }}$ is about 2500 volts.

The range of values which could theoretically be used for $\mu, D$, and $b$ is quite large. The values used here gave the best agreement with experiment, while still remaining within theoretical ranges. To be completely consistent with the assumption that $g=0.2, \mu / D$ for the calculation of $v$ should be obtained from Equation 38; it is then roughly a factor of 13 smaller than given by Equations 34 and 35 . The resulting set of curves is shown in Fig. 5 . The correct order of magnitude for the fraction recombining is given again, however the model predicts small variation in pulse height with bias in this region $(v<0.05)$. It is then implied that the detector is normally operated at a very much lower set of biases than is needed to give full signal, i.e. $E_{\text {crit }}=14,000 \mathrm{~V} / \mathrm{cm}$. 
Fig. 4. Model Prediction With $\mu$, D From Ref. 29 Versus ${ }^{3}$ He Recoil Edge Location

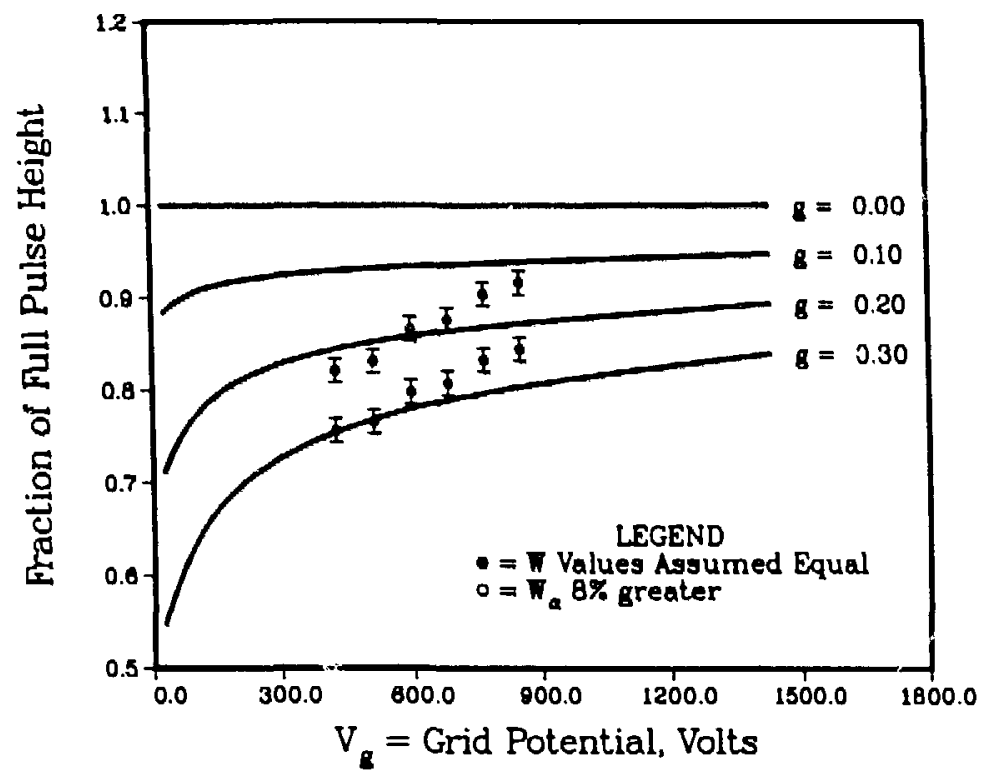

Fig. 5. Model Prediction With $D / \mu=1 \mathrm{~V}$ Versus ${ }^{3} \mathrm{He}$ Recoil Edge Location

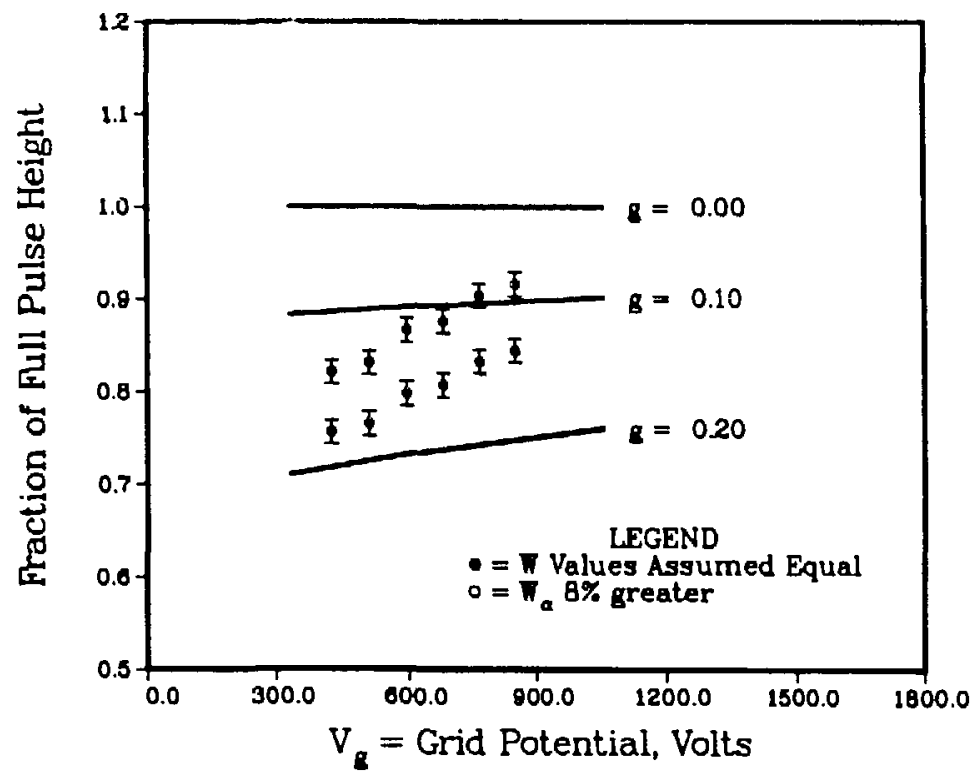


The FNS-1 detector was irradiated with "thermal" neutrons from a moderated $1 \mathrm{Ci} \mathrm{Pu}(\mathrm{Be})$ source. Pulses in the detector, due to the very large ${ }^{3} \mathrm{He}(n, p)$ cross section $(Q=764 \mathrm{KeV}$ ) are due to the $573 \mathrm{KeV}$ proton and $191 \mathrm{KeV}$ triton formed in the reaction. The average value of the stopping power along these tracks is about $30 \%$ that of the $1.9 \mathrm{MeV}{ }^{3} \mathrm{He}$ nucleus in the above experiment. A very similar electronics setup was used during this experiment, with the same voltage settings. An IBM PC computer was used as a multi-channel analyzer, with a simple program for determining peak centroids. An energy scale was assigned to the data: the centroid for the highest voltage settings was assigned $764.00 \mathrm{KeV}$. The results are given in Table 2. Over $10^{5}$ counts in each peak were obtained. The reproducibility of the peaks was found to be $\pm 0.1 \mathrm{KeV}$.

Table 2. Variation in Thermal Peak Centroid with Applied Potentials.

$\begin{array}{lll}\begin{array}{l}\text { Grid } \\ \text { Voltage, } \Phi_{g}\end{array} & \begin{array}{l}\text { Anode } \\ \text { Voltage, } \Phi_{a}\end{array} & \begin{array}{l}\text { Centroid } \\ \mathrm{KeV}\end{array} \\ 850 & 3000 & 764.00 \\ 765 & 2700 & 762.1 \\ 680 & 2400 & 759.9 \\ 595 & 2100 & 757.0 \\ 510 & 1800 & 753.7 \\ 425 & 1500 & 747.0 \\ 340 & 1200 & 740.0\end{array}$

The extrapolated "full" pulse height (peak centroid expected when no recombination occurs) was estimated by plotting the centroid energy against the reciprocal of the anode voltage, and then extrapolating to zero on the abscissa. The curve was found to be a rather straight line. The full pulse height was found to be located at $779.3 \mathrm{KeV}, 2 \%$ higher than that at the $\Phi_{g}=$ $850 \mathrm{~V}$ settings, indicating that $2 \%$ recombination is occurring in the thermal peak during normal operation. This figure is not unreasonable, but considering the lack of rigor, an uncertainty of $\pm 1 \%$, or $8 \mathrm{keV}$ is assigned to this number. 
The results are plotted with the model calculations in Fig. 6 . The assumptions used to calculate $v$ are the same as in Equations $34-36$. The values of $g$ shown correspond to those in the ${ }^{3} \mathrm{He}$ recoil case, multiplied by 30\%. The results are consistent with those for the ${ }^{3} \mathrm{He}$ recoil case, with the assumption $W_{\alpha}=1.08 \times W_{\text {proton }}$ and many of the same commerits apply. The data is bracketed by $0.03<g<0.09$. A strict proportic laiity between $d E / d x$ and $f_{r}$ would require a $2-3$ times greater proton/triton loss to recombination than is observed here. The slopes of the model curves are not steep enough.

\section{Variation Fraction Recombined with Particle Energy}

In Ref. I the fractional shift leftward in the pulse height spectrum of the ${ }^{3} \mathrm{He}$ recoil edge versus its kinematically expected value was given as a function of neutron energy, and was stated as an average fraction recombined. The fraction was seen to increase sharp?y with decreasing neutron energy, a trend which was attributed to the increasing stopping power for the slower ${ }^{3} \mathrm{He}$ nuclei. The model developed here is plotted against the data from Ref. 1, with the assumptions stated in Eqs. 36 - 40 in Fig. 7 . The horizontal axis is the maximum ${ }^{3} \mathrm{He}$ recoil energy at a given neutron energy. Also shown in the plot is the average stopping power along the ${ }^{3} \mathrm{He}$ track, normalized to equal 0.10 at $E=2 \mathrm{MeV}$. The trend in the stopping power and the model calculations are not monotonic; the stopping power has a maximum value at $E$ $=800 \mathrm{KeV}$. The decrease in the fraction recombined is much sharper than the maximum slope of the $d E / d x$ curve.

The variation at the lowest energies must be due to factors which cannot be easily modeled, e.g. a cooler electron spectrum. At $E>1 \mathrm{MeV}$, thougn, a resonable adjustment to make to the calculations is to have the column radius b vary with energy. From Ref. 28 , it is seen that the electron impact ionization cross section for electron energies expected during the primary processes of ionization varies roughly inversely with the square root of the energy. Using this as a basis, the value of $b$ was taken as $b=k \sqrt{E}$, where $E$ is the ${ }^{3} \mathrm{He}$ energy, and $k=2 \times 10^{-4} \mathrm{~cm}$. The values of $\mu$ and $D$ were taken from Eqs. 34 and 35. The model calculations are then shown in Fig. 8. Reasonable values for the electron temperature give the correct trend for the fraction recombined at higher energies. 

Peak Location in Thermal Neutron Experiment

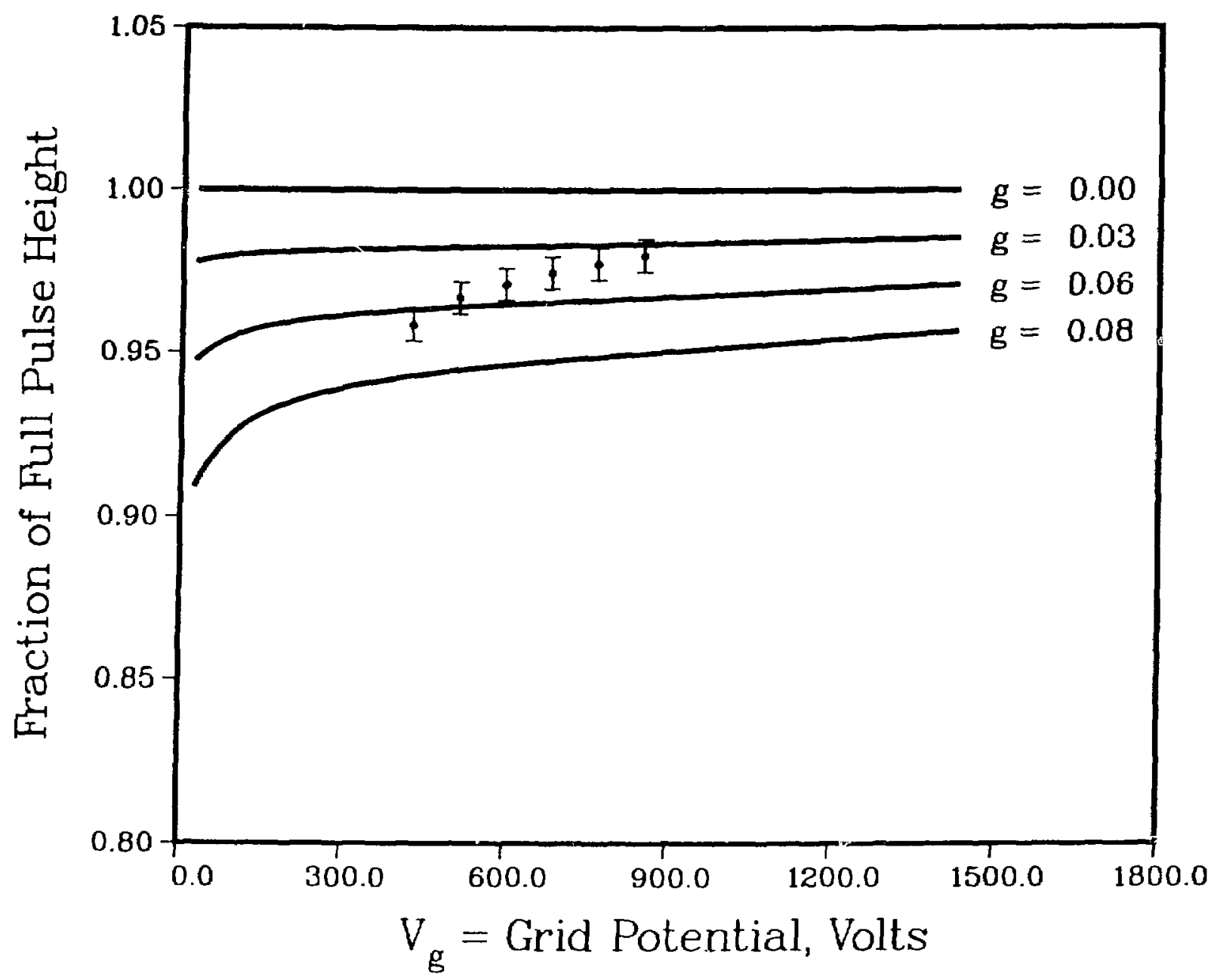


Fig. 7. Comparison of Model With Data of Ohm, et al, Fig 10

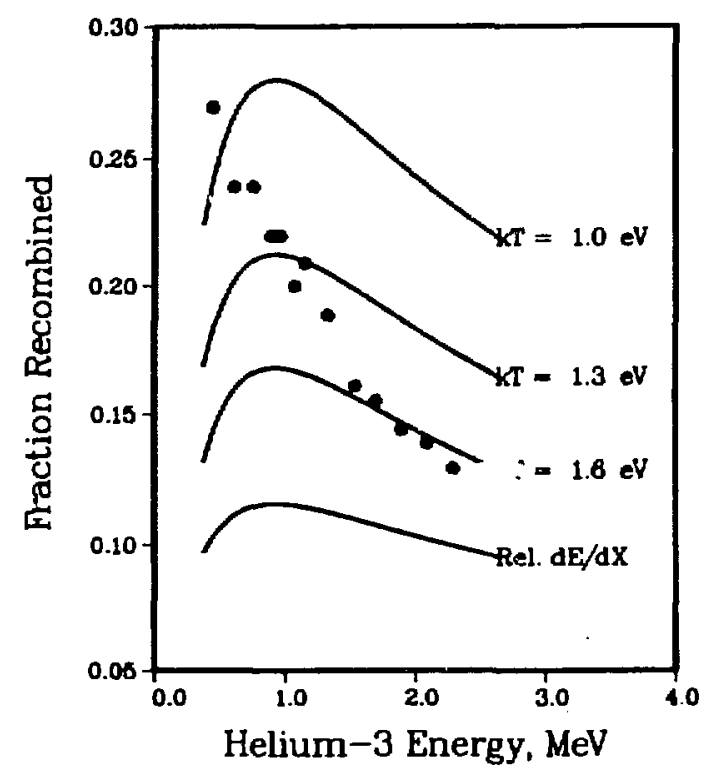

Fig. 8. Comparison of Model With Data of Ohm, et al, Fig 10 $\mu$ and $D$ from Ref. 29 , and $b=k \sqrt{ } E$

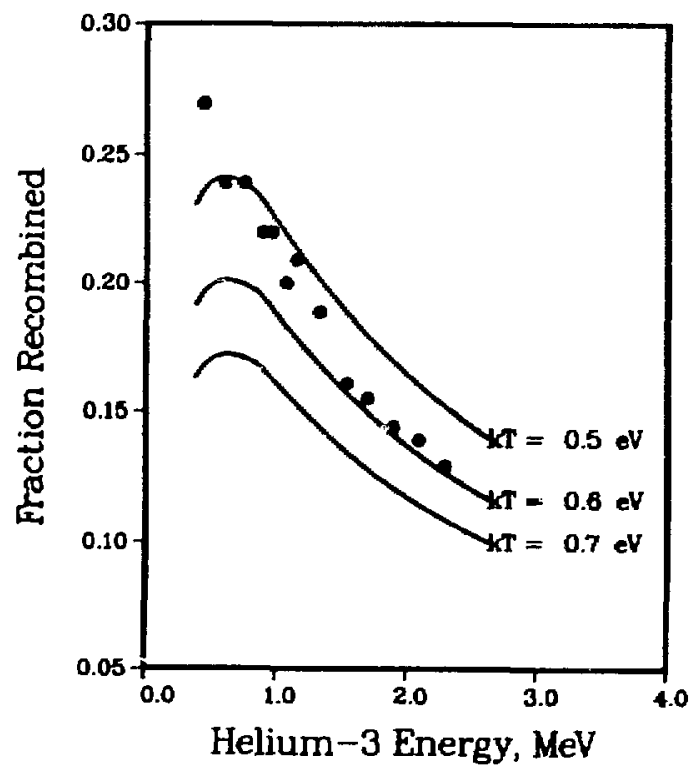




\section{Conclusions}

The model predicts the fractional recombination rates, both for ${ }^{3} \mathrm{He}$ nuclei and protons/tritons assuming an electron temperature during recombination of about $1 \mathrm{eV}$. It also predicts a variation in pulse height with applied potential; however the variation seen in experiment is somewhat stronger. The model seems to be limited by lack of higher order terms in the coupling of the continuity equation with Poisson's equation, and also lack of an a-priori method for calculating $k T$ and $b$ over a wide range of particle energies. For detectors operated above the critical field, with $g<1$, it is predicted that space charge effects are relatively unimportant and a Jaffe-like model can be taken to apply.

The performance of a detector will be adversely affected by recombination with increasing pressure. Eventually, the space charge effects will dominate and cause signal loss at a rate that increases faster than the pressure. The experiments indicated an average $2 \%$ recombination in the FNS- 1 detector thermal peak; if this varies by $\pm 0.5 \%$ with the particle track position in the detector there will be a $17 \mathrm{KeV}$ rontribution to FWHM for $1 \mathrm{MeV}$ neutrons (or $7 \mathrm{KeV}$ for thermals), which is a large fraction of the experimental $20 \mathrm{KeV}$ resolution of the detector.

Loss to recombination. De lowered by raising the electric field above the critical value, which was found in this model to be the same (for moderate values of "g") as in the Jaffe model. A quencher species added to the gas will increase the drift speed but also increase the Langevin recombination coefficient.

\section{References}

1. H. Ohm, K.-L. Kratz, and S. G. Prussin, Nucl. Inst. Meth. A256 (1987) 76.

2. W. C. Sailor, S. G. Prussin, and M. Derzon, "Monte Carlo Calculation of the Response Function for a "He Neutron Spectrometer" (to be published in Nuclear Instruments and Methods in Physics Research, Section A).

3. Seforad Applied Radiation Labs. Spectrometer Manual, Emek Hayarden, Israel. 
4. P. R. Tunnicliffe and A. G. Ward, Proc. Phys. Soc. A65 (1952) 233.

5. R. Batchelcr, R. Aves, and T. H. R. Skyrme, Rev. Sci. Inst. 26 (1955) 1037.

6. B. M. Isaev, I. V. Gordeev, Yu. I. Bregadze, Yu. S. Gerasimov, and G. N. Antipenkova, Meas. Tech. R. 67 (1967) 389.

7. G. Jaffe, Ann. d. Physik IV, Vol. 42 (1938), pp. 303-344.

8. J. W. Boag, in F. H. Attix and W. C. Roesch, Radiation Dosimetry, 2nd ed., Academic Press, 1966.

9. W. Hofmann, U. Klein, M. Schulz, J. Spengler and D. Wegener, Nucl. Inst. Meth. 135 (1976) 151-156.

10. $\therefore$ Prunier, R. Allemand, M Laval and G. Thomas, Nucl. Inst. Meth. 109 (1973) $257-264$.

11. T. Lindblad, L. Bagge, A. Engstrom, J. Bialkowski, et.al., Niscl. Inst. Meth. 215 (1983) 183-191.

12. C. Brassard, Nucl. Inst. Meth. $162(1979)$ 29-47.

13. E. Shibamura, A. Hitachi, T. Doke, T. Takahashi, et.ai., Nucl. Inst. Meth. $131(1975) 249-258$.

14. Schott, M., Molecular Crystals and Liquid Crystals, 10 (1970) 399.

15. D. B. Brown and C. M. Dozier, IEEE Trans. Nucl. Sci., NS-28 (1981) 4142.

16. T. R. Oldham and J. M. McGarrity, IEEE Trans. Nucl. Sci., NS-28 (1981) 3975.

17. R. W. Tallon, W. T. Kemp, M. R. Ackermann, M. H. Owen, and A. H. Hoffland, IEEE Trans Nucl. Sci. NS-34 (1987) 1208.

18. D. E. Lea, Proc. Cambridge Phil. Soc., Vol. 30 (1934) 80-101. 
19. H. A. Kramers, Physica XVIII, No, 10 (1952) 665.

20. G. C. Messenger, IEEE Trans. on Nucl. Sci., NS-28, (1982) 2024.

21. J. B. A. Mitchell and J. W. McGowan, in F. Brouillard and J. W. McGowan, Physics of Ion-Ion and Electron-Ion Collisions, Plenum Press, 1983.

22. H. S. W. Massey, Adv, in Phys. Vol. 1, (1952) 395-426.

23. D. R. Bates in M. R. C. MCDowell, E. W. MCDaniel, eds., Case Studies in Atamic Physics, Vol. 4, North Holland, Amsterdam, (1974) 57.

24. D. A. Armstrong, E. S. Sennhauser, J. M. Warman, and V. Sowada, Chem. Phys. Letters $86(1982) 281$.

25. J. M. Warman, E. S. Sennhauser, and D. A. Armstrong, J. Chem. Phys. 70 (1979) 995.

26. E. S. Sennhauser, D. A. Armstrong, and J. M. Warman, Radiat. Phys. Chem. $15(1980) 479$.

27. D. R. Bates, J. Phys. B 14 (1981) 3525.

28. L. Christophorou, Atomic and Molecular Radiation Physics, Wiley, London, 1971.

29. V. Palladino and B. Sadoulet, Nucl. Inst. and Meth. 128 (1975) 323.

30. C. W. Duncan and I. C. Walker, J. Chem. Soc. Faraday Trans. 2 (1972)

$1514-1521$. 